\title{
Soluble forms of the adhesion molecule E-cadherin in urine
}

\author{
R E Banks, W H Porter, P Whelan, P H Smith, P J Selby
}

\begin{abstract}
The adhesion molecule E-cadherin is essential for maintaining epithelial intercellular adhesion. Loss or reduced expression of E-cadherin has been related to invasive behaviour in a wide range of carcinomas. Using immunoblotting techniques, the existence of multiple soluble forms of E-cadherin was demonstrated in urine from healthy volunteers and patients with benign urinary tract disorders or bladder cancer. The existence of soluble forms of E-cadherin in the urine may reflect shedding from the urinary tract epithelium as part of the normal turnover of this molecule. The possibility that enhanced shedding may contribute to the loss of E-cadherin expression/function in malignancy is discussed

(f Clin Pathol 1995;48:179-180)
\end{abstract}

Keywords: Bladder cancer, urine, E-cadherin, adhesion molecules.

Cellular adhesion molecules may play an important role in tumour invasion and metastasis. Study of these molecules is important for understanding tumour biology but also may potentially be of use in the diagnosis and prognostic evaluation of cancers. The cadherins, $\mathrm{Ca}^{2+}$ dependent homotypic cell-cell adhesion molecules, are essential for establishing and maintaining intercellular connections. Ecadherin (120 kilodaltons) is involved in epithelial cell-cell interactions, with loss or reduced expression often correlating with invasiveness and dedifferentiation in a wide range of human cancers. ${ }^{1}$ Other mechanisms may also contribute to impairment of the adhesive function of E-cadherin-for example, point mutations in the calcium binding sites, ${ }^{2}$ loss of the cadherin associated catenins which form the link with the cytoskeleton, ${ }^{3}$ or posttranslational modifications such as proteolysis. ${ }^{4}$ Soluble forms of several adhesion molecules have been described ${ }^{5}$ and an 80 kilodalton fragment of E-cadherin has been reported to be shed by the MCF-7 human breast cancer cell line. ${ }^{67}$ Similar fragments have recently been described in serum samples from healthy volunteers with higher levels present in patients with cancer. ${ }^{8}$ The purpose of this study was to investigate whether soluble forms of E-cadherin are normally present in urine and whether such forms are present in benign and malignant urinary tract disorders.

\section{Methods}

Urine samples from 28 patients and 14 normal healthy donors (table) were collected into vials containing $100 \mathrm{KIU} / \mathrm{ml}$ of the proteinase inhibitor aprotinin, $10 \mathrm{mM}$ HEPES buffer $(\mathrm{pH}$ $7 \cdot 6$ ), $50 \mathrm{IU} / \mathrm{ml}$ penicillin, and $50 \mu \mathrm{g} / \mathrm{ml}$ streptomycin (final concentrations in urine). Samples were processed within one hour of collection. Following centrifugation to remove cellular material, urine samples were dialysed, concentrated ("Centricon", Amicon, Stonehouse, $\mathrm{UK}$ ) and stored at $-70^{\circ} \mathrm{C}$ until analysis.

All samples were analysed by electrophoresis on $8 \%$ sodium dodecyl sulphate (SDS) polyacrylamide gels under reducing conditions. The volume of urine sample loaded was adjusted on the basis of creatinine concentration to correct for renal concentration effects, with the equivalent of $40 \mathrm{nmoles}$ of creatinine being loaded per sample well. Following electrophoresis, gels were electroblotted onto nitrocellulose membranes ("Hybond C Super", Amersham, Little Chalfont, UK). Blots were blocked with TRIS buffered saline $/ 0 \cdot 1 \%$ Tween 20 containing $10 \%(\mathrm{w} / \mathrm{v})$ dried skimmed milk. E-cadherin was detected by sequential incubations with monoclonal anti-E-cadherin antibody ${ }^{9}$ HECD-1, $1 \mu \mathrm{g} / \mathrm{ml}$ ) or mouse $\mathrm{IgG}_{1}$ as an irrelevant control, biotinylated $\mathrm{F}(\mathrm{ab})_{2}$ rabbit antimouse immunoglobulins (Dako, High Wycombe, UK) and peroxidase conjugated Streptavidin (Dako). Blots were incubated in enhanced chemiluminescent reagent ("ECL system", Amersham) with subsequent detection of light emitted using XAR5 film (Kodak, Rochester, USA).

\section{Results}

A main E-cadherin band of 80 to 85 kilodaltons was detected in all but four of the samples with a further additional band of 65 to 70 kilodaltons apparent in approximately $20 \%$ of control samples and $50 \%$ of patient samples (table). A single band of approximately 35 kilodaltons was seen in a sample from a patient who had undergone a kidney/pancreas transplant in whom the pancreatic duct drained directly into the bladder. No bands were detected in the blots incubated with irrelevant primary antibody as a non-specific binding control. Although only semiquantitative, it is apparent (figure) that when normalised for creatinine concentrations, considerable variation in amounts of E-cadherin can be seen between urine samples. Most urine samples from patients with G2/3 tumours (particularly those with invasive tumours) appeared to contain more E-cadherin than samples from the healthy controls or patients with benign urinary tract disorders, although an overlap between groups was seen. 
Details of the groups from whom urine samples were obtained and the forms of soluble Ecadherin detected by immunoblotting.

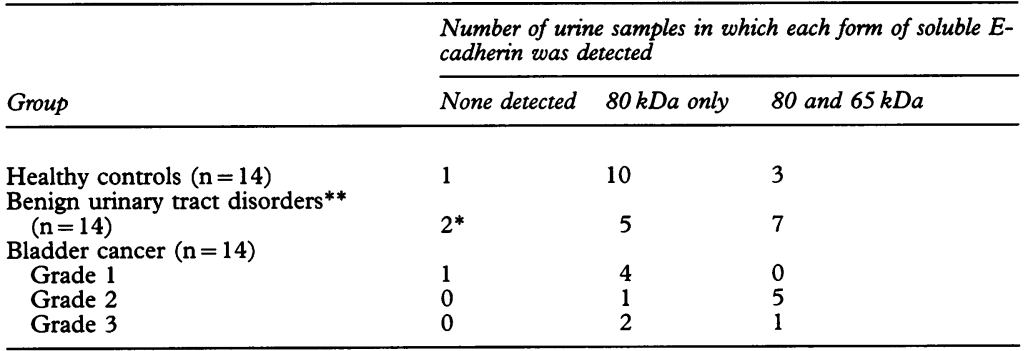

* One of these samples contained a 35 kilodalton band.

**Post-renal transplant, urinary tract infection, bladder inflammation/dysplasia.

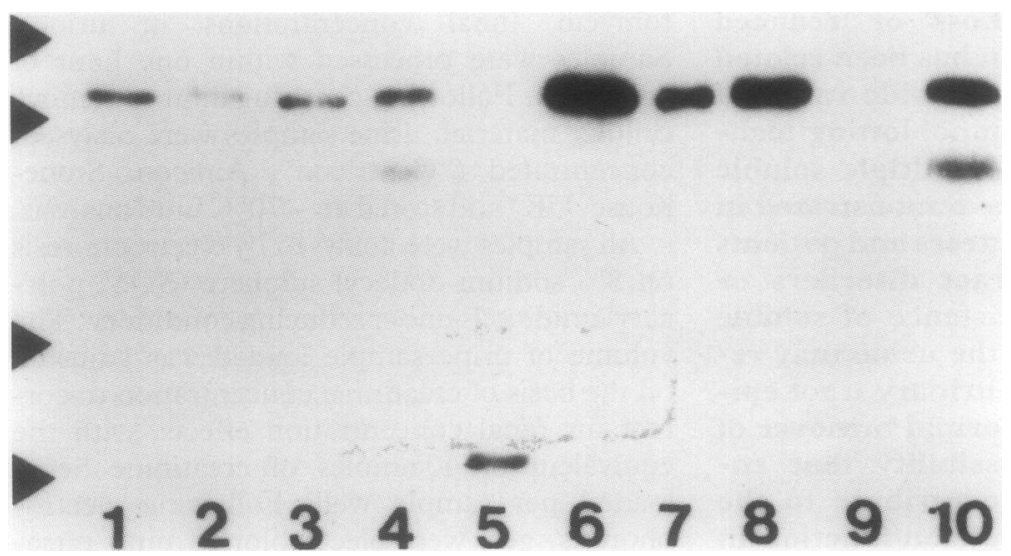

Immunoblot analysis of $E$-cadherin in urine samples (equivalent of 40 nmoles creatinine per lane). Arrows indicate the position of molecular weight markers at 97,66, 45, and 31 kilodaltons. Lanes 1 and 2, normal volunteers; lane 3, urinary tract infection; lane 4, post-kidney transplant; lane 5, post-kidney/pancreas transplant; lanes 6 to 8, invasive $G 2$ bladder cancer ( $p T 1 / p T 2)$; lane 9, G1 bladder cancer ( $p T a)$; lane 10, non-invasive G2 bladder cancer ( $p T a)$.
Decreased expression of E-cadherin has been detected immunohistochemically in many different tumour types ${ }^{1}$ and in bladder tumours has been reported to correlate with poor survival. $^{10}$ Our study demonstrates that concentrations of urinary E-cadherin may be increased in some patients with bladder cancer, particularly in those with higher grade invasive tumours, although numbers are limited. These findings, together with the quantitative increases in E-cadherin found in the serum of some patients with cancer, ${ }^{8}$ suggest that increased proteolytic activity seen in tumours may contribute to the release of the soluble form. Thus, increased shedding may, at least in part, contribute to the decreased cellular expression of E-cadherin seen in tumour progression.

A larger prospective study examining urinary E-cadherin with a quantitative immunoassay combined with immunohistochemistry of biopsy specimens is currently being undertaken to clarify the relevance of shedding of E-cadherin in tumour pathology, the mechanism of shedding and the possible clinical use of such measurements.

This study was supported by the Imperial Cancer Research Fund. We are very grateful to Professor $M$ Takeichi of Kyoto University, Japan, for his generous gift of the HECD-1 antibody, to Dr A Davison for additional clinical data, to Mrs C Brook for help in sample handling and to $\mathrm{Mr} \mathrm{M}$ Bosomworth for creatipine measurements $\mathbb{W}$ H Porter is on sabbatical leave Dathology and Laboratory Medicine, University of Kentucky Medical Centre, Lexington, Kentucky, USA.

1 Takeichi M. Cadherins in cancer: implications for invasion and metastasis. Curr Opin Cell Biol 1993;5:806-11.

2 Ozawa M, Engel J, Kemler R. Single amino acid substitutions in one $\mathrm{Ca}^{2+}$ binding site of uvomorulin abolish stitutions in one Ca

These preliminary studies show that soluble forms of E-cadherin are present in variable amounts in urine and suggest that future quantitative studies may reveal a role for soluble E-cadherin in the diagnosis and prognosis of invasive disease. The molecular weight of the main form of E-cadherin found in urine ( 80 to 85 kilodaltons) is entirely consistent with the soluble fragment of E-cadherin shed by cell lines ${ }^{67}$ and that found in serum. ${ }^{8}$ With this molecular weight it is unlikely that the $\mathrm{E}$ cadherin in the urine has been filtered from the bloodstream by the kidney and therefore it is probably derived from the renal and/or urinary tract epithelium. The significance of the smaller fragments is not yet clear but their presence may reflect partial proteolytic degradation of the 80 kilodalton form, particularly in the case of the 35 kilodalton form in the sample taken following the pancreatic/renal transplant where pancreatic secretions drained directly into the bladder.
Shimoyama Y, Nagafuchi A, Fujita S, Gotoh M, Takeichi $\mathrm{M}$, Tsukita S, et al. Cadherin dsyfunction in a human cancer cell line: possible involvement of loss of $\alpha$-catenin expression in reduced cell-cell adhesiveness. Cancer Res 1992;52:5770-4.

4 Matsuura K, Kawanishi J, Fujii S, Imamura M, Hirano S, Takeichi $M$, et al. Altered expression of E-cadherin in gastric cancer tissues and carcinomatous fluid. Brf Cancer 1992;66:1122-30.

5 Gearing AJH, Newman W. Circulating adhesion molecules in disease. Immunol Today 1993;14:506-12.

6 Damsky CH, Richa J, Solter D, Knudsen K, Buck CA Identification and purification of a cell surface glycoprotein mediating intercellular adhesion in embryonic and adult tissue. Cell 1983;34:455-66.

7 Wheelock MJ, Buck CA, Bechtol KB, Damsky CH. Soluble $80-\mathrm{kd}$ fragment of cell-CAM 120/80 disrupts cell-cell adhesion. $\mathcal{F}$ Cell Biochem 1987;34:187-202. $M$, Kato I. Soluble E-cadherin fragments increased in

9 Shimoyama Y, Hirohashi S, Hirano S, Noguchi $M$ Shimosato Y, Takeichi $M$, et al. Cadherin cell-adhesion molecules in human epithelial tissues and carcinomas. Cancer Res 1989;49:2128-33.

10 Bringuier PP, Umbas R, Schaafsma HE, Karthaus HFM, Debruyne FMJ, Schalken JA. Decreased E-cadherin immunoreactivity correlates with poor survival in patients munoreactivity correlates with poor survival in pati
8 Katayama M, Hirai S, Kamihagi K, Nakagawa K, Yasumoto circulation of cancer patients. Br F Cancer 1994;69:580-5. 